\title{
Anticuerpos IgG4 específicos anti Aedes aegypti como factor protector en niños con dengue grave
}

\author{
Claudia Lorena García a Dalgys Martínez ${ }^{\mathbf{b}}$ - Dolly Castro ${ }^{\mathrm{a}}$ - Doris Martha Salgadoc \\ - Carlos Narváez ${ }^{c}$ - Leonardo Puertac - Jairo Antonio Rodríguezc
}

\begin{abstract}
Resumen: Introducción: la infección por el virus del dengue es un problema de salud pública mundial. El virus es transmitido por la picadura de mosquitos del género Aedes. Las proteínas de la saliva del vector Aedes aegypti inducen anticuerpos IgE e IgG4 específicos, cuya relación con la gravedad del dengue aún es desconocida. Objetivo: evaluar la asociación entre anticuerpos IgE e IgG4 específicos anti $\boldsymbol{A}$. aegypti con la gravedad de la infección por dengue. Método: se realizó un estudio transversal en el que se incluyeron 16 niños con dengue grave (DG), 15 niños con dengue con signos de alarma (DCSA) y 26 niños sanos, todos menores de 15 años. Se determinaron niveles séricos de IgE e IgG4 específicas de $A$. aegypti; también se cuantificó VEGF, SST2 y VEGFR1 por ELISA. Para las variables cualitativas se calcularon proporciones y odds ratio (OR); en las variables cuantitativas se hallaron medianas, rango intercuartílico y se utilizó la prueba U Mann Whitney. Resultados: la oportunidad de los niños de tener DG con niveles séricos de IgG4 específica mayores de 0,5 OD es $78 \%$ menor [OR=0,22] (IC de $95 \%$ de $0,06-0,77)$, comparado con la oportunidad de tener DG con niveles séricos de IgG4 específica menores de 0,5 OD. Plaquetas $(p=0,0002)$ y VEFG $(p=0,003)$ más elevado en los pacientes con DCSA y sST2 fue más alto en el DG ( $p=0,004)$. Conclusión: niveles de anticuerpos de IgG4 anti $A$. aegypti se relacionan con menor gravedad clínica del dengue.
\end{abstract}

Palabras clave: dengue; inmunoglobulina E, inmunoglobulina G4; alergia al mosquito Aedes aegypti; tropomiosina

Fecha de recepción: 3 de julio de 2018 Fecha de aprobación: 30 de mayo de 2019

Cómo citar: García Rojas CL, Martínez D, Castro D, Salgado DM, Narváez C, Puerta L, Rodríguez JA. Anticuerpos IgG4 específicos anti Aedes aegypti como factor protector en niños con dengue grave. Revista Med. 27(2): 13-22. Disponible en: https://revistas.unimilitar.edu.co/index.php/rmed/article/view/3548

a Maestría en Epidemiología, Facultad de Salud. Universidad Surcolombiana y Universidad Antonio Nariño, Neiva, Colombia. Correo electrónico: claudia_garcia@uan.edu.co

b Instituto de Investigaciones Inmunológicas. Universidad de Cartagena, Cartagena, Colombia.

c Grupo Parasitología y Medicina Tropical, Programa de Medicina, Facultad de Salud. Universidad Surcolombiana, Neiva, Colombia. 


\title{
Aedes aegypti-specific IgG4 Antibodies as a Protection Factor in Children with Severe Dengue
}

\begin{abstract}
Introduction: Dengue virus infection is a global public health problem. The bite of Aedes mosquitoes transmits the virus. The proteins in the saliva of the Aedes aegypti vector induce specific IgE and IgG4 antibodies, whose relationship with the severity of dengue is still unknown. Aim: To evaluate the association between $A$. aegypti-specific IgE and IgG4 antibodies and the severity of dengue infection. Method: A cross-sectional study was carried out involving 16 children with severe dengue (DG), 15 children with dengue and warning signs (DCSA), and 26 healthy children, all of them under 15 years of age. Serum levels of $A$. aegypti-specific IgE and IgG4 were determined; VEGF, SST2, and VEGFR1 were also quantified by ELISA. For the qualitative variables, proportions and odds ratios (OR) were calculated; as to the quantitative variables, medians and interquartile range were found and the U Mann Whitney test was used. Results: Children's chance of having DG with specific IgG4 serum levels greater than $0.5 \mathrm{DO}$ is $78 \%$ lower [OR $=0.22$ ] ( $95 \% \mathrm{CI}, 0.06-0.77$ ), compared to the possibility of having DG with specific IgG4 serum levels less than 0.5 Do. Platelets $(p=0.0002)$ and VEFG $(p=0.003)$ that are higher in patients with DCSA and SST2 were higher in DG $(p=0.004)$. Conclusion: $A$. aegyptispecific IgG4 antibody levels are related to lower clinical severity of dengue.
\end{abstract}

Keywords: Dengue; immunoglobulin E, immunoglobulin G4; allergy to the Aedes aegypti mosquito; tropomyosin

\section{Anticorpos IgG4 específicos anti-aedes aegypti como fator de proteção em crianças com dengue grave}

Resumo: Introdução: a infecção pelo vírus da dengue é um problema mundial de saúde pública. O vírus é transmitido pela picada de mosquitos do gênero Aedes. As proteínas na saliva do vetor Aedes aegypti induzem anticorpos IgE e IgG4 específicos, cuja relação com a gravidade da dengue ainda é desconhecida. Objetivo: avaliar a associação entre anticorpos IgE e IgG4 específicos Anti-Aedes aegypti com a gravidade da infecção por dengue. Método: foi realizado um estudo transversal no qual foram incluídas 16 crianças com dengue grave (DG), 15 crianças com dengue com sinais de alarme (DCSA) e 26 crianças saudáveis, todas com menos de 15 anos de idade. Os níveis séricos de IgE e IgG4 específicos para Aedes aegypti foram determinados. VEGF, SST2 e VEGFR1 também foram quantificados por ELISA. Para as variáveis qualitativas, foram calculadas proporções e odds ratio (OR). Nas variáveis quantitativas foram encontradas medianas, intervalo interquartil e utilizado o teste $U$ de Mann Whitney. Resultados: a chance de as crianças terem DG com níveis séricos de IgG4 específica maiores que 0,5 OD é 78\% menor [OR=0,22] (IC 95\% 0,06-0,77), em comparação com a chance delas terem DG com níveis séricos de IgG4 específica menor que 0,5 OD. As plaquetas $(p=0,0002)$ e VEFG $(p=0,003)$ foram maiores nos pacientes com DCSA e o SST2 foi maior no DG $(p=0,004)$. Conclusão: os níveis de anticorpos IgG4 Anti-Aedes aegypti estão relacionados à menor gravidade clínica da dengue.

Palavras-chave: dengue; imunoglobulina E; imunoglobulina G4; alergia ao mosquito Aedes aegypti; tropomiosina 


\section{Introducción}

La infección por el virus del dengue (DENV) es una enfermedad de carácter endemoepidémico, transmitida por mosquitos del género Aedes, principalmente por A. aegypti. En los últimos cincuenta años, la incidencia de esta esta enfermedad ha aumentado a nivel mundial (1). Anualmente, se presentan entre 50 y 100 millones de infecciones sintomáticas, de las cuales 500000 son dengue grave (DG) y, de estas, el $2,5 \%$ tiene desenlace fatal (2).

En Colombia el dengue representa un problema prioritario en salud pública, por múltiples factores, incluyendo la reemergencia e intensa transmisión viral, con tendencia creciente, y circulación de los serotipos DENV-1, DENV-2, DENV-3 y DENV-4 (3). En 2016, se notificaron 103822 casos de dengue; 61220 casos (50\%) sin signos de alarma; $41555 \mathrm{ca}$ sos $(40 \%)$ de dengue con signos de alarma (DCSA) y 1047 casos $(1,0 \%)$ de DG. El 25,1\% de los casos de DG se presentó en menores de 15 años. En el departamento colombiano del Huila, la incidencia fue de 488 casos por cada 100000 habitantes en riesgo; datos por los cuales se ubica por encima de la media nacional (4).

Por otro lado, Colombia presenta una prevalencia de alergias del 12,0\% en la población de niños en edad escolar, que suele ser la población más afectada; las alergias respiratorias (asma y rinitis) son las más frecuentes y, en menor proporción, las relacionadas con la piel (5). Reportes previos mostraron que personas con hipertensión arterial, alergias en la piel (6) y comorbilidades como la diabetes (7) presentan mayor riesgo de desarrollar dengue hemorrágico (según clasificación Organización Mundial de la Salud [oms] - 1997). En los niños menores de 15 años infectados por DENV, el 19,54\% podría desarrollar dengue hemorrágico (6).

Además, se han encontrado niveles más elevados de IgE total en el suero de personas con niveles de IgM positivos para dengue, en comparación con individuos IgM negativos; la concentración de $\operatorname{IgE}$ fue mayor en individuos con infección primaria o secundaria de dengue, en comparación con los de individuos sin contacto previo con el DENV $(8,9)$.

Se observa clínicamente que pacientes con dengue tienen manifestaciones dérmicas como eritema y prurito, lo que sugiere degranulación del mastocito, síntomas que mejoran con antihistamínicos. El DENV es capaz de desencadenar la degranulación del mastocito e, incluso, se ha observado que los mastocitos son blanco del DENV por vía dérmica (10).

En su proceso de alimentación, antes de succionar la sangre, los mosquitos depositan proteínas de la saliva (11), como tropomiosinas, glucosidasas antibacterianas y anticoagulantes que podrían interaccionar con la IgE sobre la superficie del mastocito, al cual degranulan en el proceso. El factor antiagregante plaquetario (PAF) y algunas citoquinas con efecto endotelial, como el factor de crecimiento endotelial vascular (VEGF) también son producto de la degranulación del mastocito; generan vasodilatación y están relacionadas con la gravedad del dengue.

En pacientes alérgicos, las proteínas provenientes de la saliva del mosquito inducen IgE específica, que activa el receptor Fceri, lo que induce la liberación de mediadores preformados (12). Contrario a la IgE, parece ser que la IgG4 contra proteínas del vector actúa como inhibidor de la degranulación ya que compite con la IgE para la unión con los alergenos (13-16).

La IL-33 producida por el epitelio dérmico induce LT CD4 Th2, a través de su interacción con el receptor sт2. Sin embargo, la forma soluble (ssT2) de este receptor es capaz de antagonizar la IL-33. El sst2 se ha visto incrementado en el DG por lo que su medida puede ser reflejo de la actividad del mastocito y la inducción del LTh2, el cual promueve la producción de IgE por parte de los linfocitos B $(17,18)$.

Por otro lado, anticuerpos IgE e IgG4 contra proteínas del Aedes aegypti fueron medidos para identificar si esas proteínas inducen IgG4 o IgE en niños con dengue y si este reconocimiento se asocia con la gravedad de la enfermedad $(11,14,19,20)$. Asimismo, se evalúa si existe relación entre los niveles séricos de anticuerpos IgE e IgG4 específicos contra proteínas del Aedes con biomarcadores previamente asociados con la gravedad del dengue en niños como sST2, VEGF Y VEGFR1. 


\section{Materiales y métodos}

\section{Población de estudio}

Se llevó a cabo un estudio de corte transversal en el que se incluyeron 66 niños menores de 15 años $(n=66)$ con infección por dengue, que asistieron al Hospital Universitario Hernando Moncaleano Perdomo (Neiva), durante el periodo de 2011 a 2016 (junio). Los criterios de inclusión fueron los de la clasificación revisada en 2009 por la oms (20) tanto para dengue grave (DG) como para dengue con signos de alarma (DCSA).

Para DG, se tuvieron en cuenta: niños con signos clínicos o radiológicos de derrame pleural, ascitis, hipotensión con presión arterial media menor de $70 \mathrm{~mm}$ de $\mathrm{Hg}$ o presión de pulso menor de $20 \mathrm{~mm}$ de $\mathrm{Hg}$, acompañados de recuento de plaquetas por debajo de las 100000 células $/ \mathrm{mm}^{3}$, con manifestaciones hemorrágicas o sin ellas, alteraciones del sistema nervioso central y hepatomegalia.

Por su parte, para DCSA se tuvieron en cuenta estos criterios: niños que presentan deterioro clínico con dolor abdominal intenso y continuo, vómito persistente, acumulación de líquido, hematemesis o hematuria, letargias, hepatomegalia de más de 2,0 cm y disminución progresiva de las plaquetas en al menos dos mediciones durante el seguimiento (21).

Además, se incluyó un grupo sin dengue, conformado por niños sanos, tomados como grupo control. Todos los pacientes firmaron el consentimiento informado y se obtuvo permiso para el estudio del Comité de Ética de la Universidad Surcolombiana, por medio del Acta No. 02, del 27 de mayo de 2015.

\section{Preparación del extracto de Aedes aegypti}

La obtención del extracto se realizó siguiendo el protocolo descrito por Cantillo y sus colaboradores (22). Brevemente, se realizó una mezcla 1:40 $\mathrm{P} / \mathrm{V}$ de liofilizado de cuerpo entero de mosquito A. aegypti en búfer fosfato salino (PBs), $\mathrm{pH} 7,2$ y se agitó durante toda la noche a $4{ }^{\circ} \mathrm{C}$. Posteriormente, la mezcla se centrifugó a $10000 \mathrm{rpm}$, durante 10 minutos y el sobrenadante se filtró por una membrana con poros de $0,2 \mu \mathrm{m}$. Finalmente se dializó en agua destilada en una bolsa de diálisis de punto de corte 3,500-Da (Spectra / PorDialysis Membranes, Houston, Texas, EE. UU.) y se liofilizó. La concentración de la proteína obtenida se determinó mediante el ensayo de Bradford.

\section{Ensayo de ELISA}

La medición del nivel sérico de IgE e IgG4 específicas se realizó en el laboratorio de inmunología de la Universidad de Cartagena. Brevemente, se recubrieron los pozos de microplacas con $0,5 \mu \mathrm{g}$ de extracto de mosquito $A$. aegypti en $100 \mu \mathrm{l}$ de tampón carbonato/bicarbonato 0,05 $\mathrm{M}(\mathrm{pH} 9,6)$. Después de lavar tres veces con PBs con Tween-20 (PBS-T), la unión inespecífica se bloqueó durante 1,0 hora con PBs-T que contenía albúmina sérica bovina (BSA) al 1,0\%, seguido por tres lavados. Las microplacas se incubaron después durante 2,0 horas con suero del paciente diluido 1:8 en PBS-T/BSA al 1,0\% y, después del lavado, se incubaron durante 1,0 hora anticuerpos de ratón anti-Fc de $\operatorname{IgE}$ o de IgG4 humanos, conjugados con peroxidasa de rábano diluida 1:2000 (Southern, Biotech).

Finalmente, las microplacas se lavaron e incubaron con dihidrocloruro de o-fenilendiamina (Sigma-Aldrich). La reacción se detuvo con $\mathrm{HCl}$ y se leyó la absorbancia a $492 \mathrm{~nm}$. Todas las muestras se ensayaron por duplicado. Se usaron tres desviaciones estándar por encima del valor medio de la densidad óptica (492 nm) del suero del sujeto control, para determinar el límite para la unión positiva de IgE e IgG4.

\section{Cuantificación de SST2 y VEFG en el plasma}

Para la cuantificación del ssT2 y VEFG se tomaron muestras de sangre obtenidas en la fase aguda de la enfermedad, en tubos con EDTA. Se obtuvo plasma por centrifugado y se congeló a $-70^{\circ} \mathrm{C}$ hasta su uso. Los niveles plasmáticos de ssT2 y VEFG se cuantificaron por ELISA, usando kits comerciales (R\&D systems, Número DY523 y DY3625) y siguiendo las instrucciones del fabricante. Para la estimación de concentración de citocinas (en pg/ml) se utilizó una curva de regresión. La concentración más baja en las curvas estándar de ELISA fue $31 \mathrm{pg} / \mathrm{ml}$ y 23 pg $/ \mathrm{ml}$ para SST2 y VEFG, como se describió en Guerrero y colaboradores (18). 


\section{Análisis estadístico}

Las variables cualitativas se expresaron en porcentaje, se dicotomizaron las variables continuas (inmunoglobulinas) con los siguientes puntos de corte (22): IgE total de 0,150 oD, IgE específica de 0,2 oD e IgG4 específica de 0,5 oD. Se consideraron negativos valores por debajo de los mencionados, para el cálculo de la odds ratio (OR). También se describieron variables de caracterización sociodemográfica, características clínicas y paraclínicas. Como variables cuantitativas, se calculó la mediana y rango intercuartílico y, para el análisis bivariado, se utilizó la prueba U de Mann Whitney. Por último, se hizo la regresión logística. Los datos fueron analizados en el programa Stata ${ }^{\mathrm{Tu}}$, versión 14.0.

\section{Resultados}

Al analizar las características sociodemográficas, se encontró un mayor porcentaje de niños de género masculino $(70 \%) \mathrm{y}$, en cuanto a edad, hay diferencia estadísticamente significativa entre el grupo de dengue (mediana de 6 años) y niños sanos (mediana de 12 años).

Los síntomas más frecuentes fueron: vómito (74\% de los niños), dolor abdominal (42\%) y cinco días de fiebre (41\%). Al comparar la presencia de estas características clínicas entre los grupos de DG y DCSA, el dolor abdominal presenta una diferencia estadísticamente significativa (or de 0,05; IC del $95 \%$ de $[0,0007-0,33])$, resultados que se muestran en la tabla 1.

Tabla 1. Características clínicas de los niños con dengue en fase aguda

\begin{tabular}{|c|c|c|c|c|}
\hline Síntomas & DG $(n=16)$ & $\begin{array}{c}\text { DCSA } \\
(n=15)\end{array}$ & OR & $\begin{array}{l}\text { IC del } \\
95 \%\end{array}$ \\
\hline \multicolumn{5}{|c|}{ Vómito } \\
\hline Sí & $69 \%$ (11) & $80 \%(12)$ & \multirow{2}{*}{0,55} & \multirow{2}{*}{$0,10-2,86$} \\
\hline No & $31 \%(5)$ & $20 \%(3)$ & & \\
\hline \multicolumn{5}{|c|}{ Dolor abdominal } \\
\hline Sí & $12 \%(2)$ & $73 \%$ (11) & \multirow{2}{*}{0,05} & \multirow{2}{*}{$\begin{array}{c}0,0007- \\
0,33\end{array}$} \\
\hline No & $88 \%(14)$ & $27 \%(4)$ & & \\
\hline \multicolumn{5}{|c|}{ Diarrea } \\
\hline Sí & $12 \%(2)$ & $13 \%(2)$ & \multirow{2}{*}{0,92} & \multirow{2}{*}{$0,11-7,5$} \\
\hline No & $88 \%$ (14) & $87 \%$ (13) & & \\
\hline
\end{tabular}

\begin{tabular}{|c|c|c|c|c|}
\hline Síntomas & DG (n=16) & $\begin{array}{c}\text { DCSA } \\
(\mathbf{n = 1 5})\end{array}$ & OR & $\begin{array}{c}\text { IC del } \\
\mathbf{9 5} \%\end{array}$ \\
\hline \multicolumn{5}{|c|}{ Ascitis } \\
\cline { 1 - 2 } Sí & $12 \%(2)$ & $7,0 \%(1)$ & \multirow{2}{*}{2,0} & $0,16-24$ \\
\hline No & $88 \%(14)$ & $93 \%(14)$ & & \\
\hline
\end{tabular}

Una vez comparados los recuentos plaquetarios entre los grupos de DCSA y DG, se observaron niveles más altos de plaquetas en el primer grupo $(\mathrm{p}=0,0002)$. Por su parte, la concentración de VEGF $(\mathrm{p}=0,003)$ fue más elevada en los niños con DCsA; mientras que el nivel sst2 fue más alto en el grupo de DG $(\mathrm{p}=0,004)$, tal como se detalla en la tabla 2 .

Tabla 2. Descripción de características paraclínicas de los niños con dengue en fase aguda

\begin{tabular}{|c|c|c|c|}
\hline Variable & DCSA $(n=15)$ & DG $(n=16)$ & $\begin{array}{l}\text { Prueba U } \\
\text { de Mann } \\
\text { Whitney }\end{array}$ \\
\hline Hematocrito & $\begin{array}{l}\text { Mediana: } 38 \\
{ }^{*} \text { RIC: } 35-40,3\end{array}$ & $\begin{array}{l}\text { Mediana: } 37,8 \\
{ }^{*} \text { RIC: } 36,0-41,7\end{array}$ & 0,782 \\
\hline Leucocitos & $\begin{array}{l}\text { Mediana: } \\
3300 \\
\text { *RIC: } 1900 \text { - } \\
5400\end{array}$ & $\begin{array}{c}\text { Mediana: } \\
4050,0 \\
\text { *RIC: } 2800,0- \\
8600,0\end{array}$ & 0,276 \\
\hline Plaquetas & $\begin{array}{l}\text { Mediana: } \\
58000 \\
\text { *RIC: } 48000 \text { - } \\
\quad 94000\end{array}$ & $\begin{array}{l}\text { Mediana: } \\
\quad 35000 \\
\text { "RIC: } 24250- \\
43000\end{array}$ & 0,0002 \\
\hline $\begin{array}{l}\text { Receptor de } \\
\text { TNF soluble } \\
\text { (STNFR) }\end{array}$ & $\begin{array}{l}\text { Mediana: } \\
\text { 7971,4 } \\
\text { *RIC: } 5492,5- \\
9585,4\end{array}$ & $\begin{array}{l}\text { Mediana: } \\
\text { 9624,3 } \\
\text { "RIC: } 4978,7- \\
12562,9\end{array}$ & 0,580 \\
\hline SIL2 & $\begin{array}{c}\text { Mediana: } \\
\text { 3895,3 } \\
\text { *RIC: } 3224,8- \\
5961,3 \\
\end{array}$ & $\begin{array}{c}\text { Mediana: } \\
5050,1 \\
\text { *RIC: } 2998,9- \\
6715,6\end{array}$ & 0,812 \\
\hline VEGF & $\begin{array}{l}\text { Mediana: } \\
171,4 \\
\text { *RIC: } 55,2- \\
292,3\end{array}$ & $\begin{array}{l}\text { Mediana: 53,3 } \\
{ }^{*} \text { RIC: 4,5-73,4 }\end{array}$ & 0,003 \\
\hline VEGFR2 & $\begin{array}{c}\text { Mediana: } \\
10603,4 \\
\text { *RIC: } 6957,8- \\
12708,5\end{array}$ & $\begin{array}{c}\text { Mediana: } \\
10974,7 \\
\text { "RIC: } 7187,3- \\
14376,5 \\
\end{array}$ & 0,874 \\
\hline IL6 & $\begin{array}{c}\text { Mediana: } \\
\text { 10,0 } \\
\text { "RIC: } 6,1-17,8\end{array}$ & $\begin{array}{l}\text { Mediana: 7,4 } \\
{ }^{*} \text { RIC: } 3,9-9,0\end{array}$ & 0,080 \\
\hline
\end{tabular}




\begin{tabular}{|c|c|c|c|}
\hline Variable & DCSA $(n=15)$ & $D G(n=16)$ & $\begin{array}{l}\text { Prueba U } \\
\text { de Mann } \\
\text { Whitney }\end{array}$ \\
\hline IL10 & $\begin{array}{c}\text { Mediana: } \\
49,0 \\
\text { *RIC: } 25,2- \\
105,1\end{array}$ & $\begin{array}{l}\text { Mediana: } 32,1 \\
{ }^{*} \text { RIC: } 12,4-70,2\end{array}$ & 0,289 \\
\hline IL8 & $\begin{array}{c}\text { Mediana: } \\
21,2 \\
\text { *RIC: } 19,2- \\
32,2\end{array}$ & $\begin{array}{l}\text { Mediana: } 23,4 \\
{ }^{*} \text { RIC: } 17,5-28,7\end{array}$ & 0,649 \\
\hline SST2 & $\begin{array}{l}\text { Mediana: } \\
318,6 \\
\text { *RIC: } 34,3- \\
1050,4\end{array}$ & $\begin{array}{c}\text { Mediana: } \\
2337,3 \\
\text { "RIC: } 985,9 \text { - } \\
3607,7\end{array}$ & 0,004 \\
\hline
\end{tabular}

* RIC: rango intercuartílico.
Al analizar los niveles plasmáticos de la IgG4 específica en niños con DG y en el grupo de control, se encontró una relación estadísticamente significativa (tabla 3). Teniendo en cuenta esta información se realizó una regresión logística, incluyendo los niveles séricos de IgG4 específicos, IgE específicos, IgE Total y dengue (tabla 4). Se determinó que la oportunidad de los niños de tener DG con niveles séricos de IgG4 específica mayores de 0,5 OD es $78 \%$ menor $(\mathrm{OR}=0,22)$, en comparación con la oportunidad de tener DG con niveles plasmáticos de IgG4 específica menores de 0,5 oD, cuando se ajusta por IgE específica e IgE total.

Tabla 3. Relación entre los niveles de IgE total, IgE e IgG4 específicas anti Aedes con la gravedad del dengue

\begin{tabular}{|c|c|c|c|c|c|}
\hline & Prueba & Sí $(n=16)$ & No $(n=26)$ & OR & IC del $95 \%$ \\
\hline \multirow{9}{*}{$\begin{array}{c}\text { Dengue con } \\
\text { signos de alarma } \\
(n=42)\end{array}$} & \multicolumn{5}{|c|}{ IgE total } \\
\hline & Positivo & $29 \%(12)$ & $26 \%(11)$ & \multirow{2}{*}{4,09} & \multirow{2}{*}{$0,88-21,57$} \\
\hline & Negativo & $9,0 \%(9)$ & $36 \%$ (36) & & \\
\hline & \multicolumn{5}{|c|}{ IgE específica } \\
\hline & Positivo & $2,0 \%(1)$ & $70 \%(3)$ & \multirow{2}{*}{0,511} & \multirow{2}{*}{$0,009-7,17$} \\
\hline & Negativo & $36 \%$ (15) & $55 \%(23)$ & & \\
\hline & \multicolumn{5}{|c|}{ IgG4 específica } \\
\hline & Positivo & $21 \%(9)$ & $45 \%$ (19) & \multirow{2}{*}{0,47} & \multirow{2}{*}{$0,10-2,15$} \\
\hline & Negativo & $17 \%(7)$ & $17 \%(7)$ & & \\
\hline \multirow{9}{*}{$\begin{array}{c}\text { Dengue grave } \\
\qquad(n=41)\end{array}$} & \multicolumn{5}{|c|}{ IgE total } \\
\hline & Positivo & $19 \%(8)$ & $27 \%(11)$ & \multirow{2}{*}{1,55} & \multirow{2}{*}{$0,36-6,78$} \\
\hline & Negativo & $17 \%(7)$ & $37 \%(15)$ & & \\
\hline & \multicolumn{5}{|c|}{ IgE específica } \\
\hline & Positivo & $2,0 \%$ (1) & $7,0 \%$ (3) & \multirow{2}{*}{0,54} & \multirow{2}{*}{$0,009-7,17$} \\
\hline & Negativo & $35 \%$ (14) & $56 \%(23)$ & & \\
\hline & \multicolumn{5}{|c|}{ IgG4 específica } \\
\hline & Positivo & $12 \%(5)$ & $46 \%$ (19) & \multirow{2}{*}{0,18} & \multirow{2}{*}{$0,036-0,879$} \\
\hline & Negativo & $24 \%(10)$ & $17 \%(7)$ & & \\
\hline
\end{tabular}


Tabla 4. Relación de niveles séricos de IgG4 específica, IgE específica e IgE total y presencia de dengue

\begin{tabular}{|c|c|c|c|}
\hline Variable & OR & IC del $95 \%$ & P \\
\hline IgG4 específica & 0,22 & $0,06-0,77$ & 0,018 \\
\hline IgE específica & 0,78 & $0,10-5,89$ & 0,814 \\
\hline IgE total & 3,42 & $1,00-11,70$ & 0,050 \\
\hline
\end{tabular}

\section{Discusión}

Se ha sugerido que existe una relación entre la concentración de IgE total y el dengue toda vez que se han encontrado niveles séricos mayores de $\operatorname{IgE}$ en pacientes que han tenido dengue hemorrágico (8). La IgE desencadena una respuesta de hipersensibilidad inmediata (23), uniéndose al mastocito por medio del receptor FceRI (24). Por lo cual la interacción de la IgE con antígenos de la saliva del mosquito vector del Aedes aegypti podría generar una inflamación temprana que influencie la evolución clínica del dengue en el paciente afectado. Cantillo y su equipo identificaron que la tropomiosina, proteína de la saliva del mosquito, generaba reactividad de la IgE, de esta manera señalan que podría servir para el diagnóstico de alergia causada por Aedes (22).

En el presente estudio, se exploran los niveles séricos de IgE e IgG4 específica para proteínas del mosquito, en relación con la gravedad del dengue. No se encontró relación entre los niveles séricos de IgE específica para proteínas de la saliva del mosquito (tropomiosina) con los análisis de las OR; pero se encontró evidencia estadísticamente significativa para la IgG4 específica. Cuando se comparó el grupo de niños con DG y los niños del grupo de control, se encontró que la oportunidad que tienen los niños de tener DG con niveles séricos de IgG4 específica mayores de 0,5 oD es $78 \%$ menor $(\mathrm{OR}=0,22)$, en comparación con la oportunidad de tener DG con niveles séricos de IgG4 específica menores de 0,5 oD.

La IgG4 es una subclase de la IgG, característica de tolerancia al alergeno, incluso, como antagonista de la actividad de la IgE, al competir con ella por la interacción con el alergeno: la IgG4 es probablemente inducida por las células $\mathrm{T}$ reguladoras, productoras de TGF- $\beta$, IL-10 e IL-35 $(8,25)$.
En comparación con otras subclases de IgG (IgG1, IgG2 e IgG3), la IgG4 proporciona una base estructural para la menor afinidad de unión a receptores de $F c \gamma(15)$.

James LK y su equipo evidencian dos posibles mecanismos por los cuales IgG4 inhibe la hipersensibilidad mediada por IgE: (a) al secuestrar el antígeno IgG4 puede funcionar como un anticuerpo bloqueante, evitando que la IgE se una al receptor y (b) se ha propuesto que la IgG4 coestimula el receptor de IgG inhibidor Fc $\gamma$ RIIb, que puede regular negativamente la señalización de FcerI y, a su vez, inhibir la activación de células efectoras (15).

El aumento en la síntesis de IgG4 disminuye la fijación del complemento por la vía clásica y, por ende, la síntesis de C5a activadora, igualmente, del mastocito (16). Al generar tolerancia la IgG4, podría verse implicada en la disminución de síntomas y, probablemente, desempeñaría una función importante de protección, para que la enfermedad en el huésped no ocurra.

Se identificó la gravedad del dengue según hallazgos paraclínicos en los niños diagnosticados con la enfermedad, atendidos en el Hospital Universitario Hernando Moncaleano Perdomo (Neiva). Se evaluó la relación con biomarcadores presentes en esta enfermedad y se encontraron diferencias estadísticamente significativas con las plaquetas, VEgF y sst2. Como en el estudio de Villar y sus colaboradores (26), se evaluó la presencia de biomarcadores predictores de trombocitopenia, vasodilatación y fuga capilar característicos del DG. Los mastocitos, productores de sustancias como histamina y citocinas como IL-6, 8 y 10 , VEGF, SST2, IFN $\gamma$, TNF $\alpha$ (27), son blanco del DENV, el cual genera su degranulación, proceso inductor de liberación sustancias mediadoras que aumentan la permeabilidad vascular.

El VEGF es un factor asociado con la angiogénesis y la permeabilidad vascular, por lo cual, según diversos estudios han evidenciado, es relevante determinarlo en el dengue. El vegf se fija a los receptores VEGFR1, VEGFR2 Y VEGFR3; algunos de estos receptores, como el VEGFR1, pueden ser solubles y, de ese modo, antagonizan la función del VEGF. En el DG, se ha encontrado aumento en las 
concentraciones séricas de VEGFRl y disminución de la VEGFr2 (12).

Un estudio realizado en Nueva Delhi (India) por Mohit Singla y sus colaboradores, en 2016, identificó que sustancias liberadas por la degranulación del mastocito se encuentran presentes en el DG; los análisis univariado y multivariado muestran que las características predominantes en el DG son el aumento de la producción de IL-6, 8, 10 y VEGF (28).

En Taiwán, Tseng y su equipo tomaron 53 pacientes con diagnóstico de dengue; 39 con fiebre de dengue y 14 con fiebre hemorrágica de dengue (criterios oms) y 5 pacientes con enfermedad febril no por dengue. Los investigadores observaron que, como en el presente estudio, los niveles medios de VEGF sérico en los pacientes con dengue hemorrágico $(54,6 \mathrm{pg} / \mathrm{ml} ; \mathrm{p}<0,05)$ fueron significativamente más altos que en los pacientes con dengue grave y el grupo de control. Además, los niveles de VEGF en los pacientes con dengue grave no fueron significativamente distintos de aquellos de los del grupo de control [ $\mathrm{p}=0$,17] (29). También se identificaron niveles más bajos de VEGF $(53,3 \mathrm{UI} / \mathrm{ml})$ en el DG, mientras que el VEGFR2 fue más alto (10974,7 UI/ $\mathrm{ml})$. Este resultado es consistente con los de Rattanamahaphoom y su equipo (30), quienes evidenciaron que la activación de células T específicas en el DG se asocia con la modulación de la expresión del VEGFR2, que puede contribuir al aumento de la capacidad de respuesta del vegf y así estimular o conducir a la activación endotelial y fuga plasmática. Además, los niveles de VEGF en pacientes con DF no fueron significativamente diferentes de los del grupo de control con enfermedad febril no debida al dengue.

Por su parte, Guerrero y su equipo demostraron el aumento del ssT2 en el DG (18). Asimismo el DG se correlacionó negativamente con el recuento plaquetario, coincidiendo con los resultados del presente estudio, pues, como se determinó por medio de la correlación de Spearman, el sst2 y las plaquetas presentan una correlación inversamente proporcional, estadísticamente significativa.

También el estudio de Villar y sus colaboradores, después de cuantificar el sst2 y otras citocinas en la fase aguda de la enfermedad, encontraron niveles significativamente más elevados en el DG. Una vez más, se afirma el papel esencial de la respuesta inflamatoria y regulación de las citocinas de la enfermedad y, por tanto, estos hallazgos contribuyen a identificar grupos en riesgo de desarrollar formas graves de la enfermedad (26).

En este estudio, se encontraron diferencias estadísticamente significativas entre la edad de los controles (niños sanos) y los casos de dengue (DCSA y DG), lo cual puede ser explicado porque las madres de niños de 5 años dificultan el acceso, ya que, como son niños sanos, no permiten el procedimiento de la toma de la muestra de sangre. Ramírez y su equipo establecen valores de referencia de concentraciones de IgE independientemente del sexo y la edad (31), mientras que Londoño-Rentería y su equipo encontraron una correlación positiva estadísticamente significativa entre anticuerpos IgG contra proteína D7 y el factor edad en mayores de 20 años y las condiciones de vida (11).

De acuerdo con características clínicas, la presencia del dolor abdominal fue estadísticamente significativa en términos de su relación con el DG, en lo que también este estudio coincide con otros estudios sobre manifestaciones y caracterización clínica en paciente con dengue, por ejemplo, los realizados en Catamarca, Argentina (32), y Cartagena, Colombia (33), que identifican el dolor abdominal en cerca del $70 \%$ de los pacientes con dengue.

Algunas limitaciones encontradas fueron efecto del diseño de la investigación, ya que el tipo de estudio transversal no permite establecer relaciones causales; el tamaño de muestra y el tipo de muestreo por conveniencia puede aumentar el error aleatorio, un aumento en el número de pacientes podría dar mayor claridad en los resultados.

Otro tipo de sesgo que pudo haber afectado los resultados es el sesgo de selección, al trabajar con una base de datos del grupo de investigación de parasitología y medicina tropical. En cuanto al sesgo de medición, no fue posible conocer la historia de alergias de los pacientes ni su clasificación, aspecto que debe tenerse en cuenta para indagar en un próximo estudio. También es probable que los padres pudieran no tener claro el tiempo de evolución de los signos y síntomas, pero, generalmente, estos 
datos son recolectados durante la fase febril, muy cerca del inicio del cuadro clínico, lo que podría hacer que la información fuera de mejor calidad. Sin embargo, es importante tener en cuenta que los registros dados en la base de datos provienen de la información registrada en la historia clínica por los residentes de la especialidad en pediatría y los reportes del laboratorio de I \& I de la Universidad Surcolombiana.

Para tener mayor certeza podría tomarse una cohorte de individuos alérgicos al mosquito y hacerles seguimiento para saber si, en caso de ser infectados con el virus dengue, presentan formas graves de la enfermedad.

\section{Agradecimientos}

Agradecemos a la Universidad Surcolombiana y a Colciencias por la financiación del estudio y al Doctor Enrique Fernández (Caldas) por donar liofilizado de A. aegypti para realizar los extractos.

\section{Referencias}

1. Verhagen LM, de Groot R. Dengue in children. J Infect. 2014; 69(S1):S77-S86. Doi: https://doi.org/10.1016/j. jinf.2014.07.020

2. Bachal R, Alagarasu K, Singh A, Salunke A, Shah P, Cecilia D. Higher levels of dengue-virus-specific IgG and IgA during pre-defervescence associated with primary dengue hemorrhagic fever. Arch Virol. 2015; 160(10):2435-43. Doi: https://doi.org/10.1007/s00705$015-2519-7$

3. Ministerio de Salud y de la Protección Social. Guía para la atención clínica integral del paciente con dengue [Internet]. 2010. [Consultado el 20 de abril de 2017]. Disponible en https://www.minsalud. gov.co/Documentos\%20y\%20Publicaciones/Gu\%C3\%ADa\%20para\%20la\%20atenci\%C3\%B3n\%20cl\%C3\%ADnica\%20integral\%20del\%20paciente $\% 20$ con $\%$ 20dengue.pdf.

4. Instituto Nacional de Salud. Boletín Epidemiológico semana-52. [Internet]. 2016. [Consultado el 7 de marzo de 2017]. Disponible en https://www.ins.gov.co/buscador-eventos/BoletinEpidemiologico/2016\%20Bolet\%C3\%ADn\%20epidemiol\%C3\%B3gico\%20semana\% 2052\%20-.pdf

5. Bissinger I, Bareno J. Clinical profile of sensitization to fungi in Medellin, Colombia. Rev Alerg Mex. 2016; 63(2):123-34.
6. Teixeira MG, Paixao Es, Costa MC, Cunha Rv, Pamplona L, Dias JP et al. Arterial hypertension and skin allergy are risk factors for progression from dengue to dengue hemorrhagic fever: a case control study. PLoS Negl Trop Dis. 2015; 9(5):e0003812. Doi: https:/doi. org/10.1371/journal.pntd.0003812

7. Teixeira Mg, Paixao Es, Costa MC, Cunha Rv, Pamplona L, Dias JP et al. Arterial hypertension and skin allergy are risk factors for progression from dengue to dengue hemorrhagic fever: a case control study. PLoS Negl Trop Dis. 2015; 9(5):e0003812. Doi: https:/doi. org/10.1371/journal.pntd.0003812

8. Míguez-Burbano MJ, Jaramillo CA, Palmer CJ, Shor-Posner G, Velásquez Ls, Lai H, Baum мк. Total immunoglobulin E levels and dengue infection on San Andres Island, Colombia. Clin Diagn Lab Immunol. 1999; 6(4):624-6.

9. Vazquez S, Lozano C, Perez AB, Castellanos Y, Ruiz D, Calzada N, Guzmán MG. Dengue specific immunoglobulins $\mathrm{M}, \mathrm{A}$, and $\mathrm{E}$ in primary and secondary dengue 4 infected Salvadorian children. J Med Virol. 2014; 86(9):1576-83. Doi: https://doi.org/10.1002/jmv.23833

10. Troupin A, Shirley D, Londono-Renteria B, Watson AM, McHale C, Hall A, Hartstone-Rose A, Klimstra wb, Gomez G, Colpitts тм. A Role for human skin mast cells in dengue virus infection and systemic spread. J Immunol. 2016; 197(11):4382-91. Doi: https://doi.org/10.4049/ jimmunol.1600846

11. Londono-Renteria BL, Shakeri H, Rozo-Lopez P, Conway mJ, Duggan N, Jaberi-Douraki M, Colpitts тм. Serosurvey of human antibodies recognizing Aedes aegypti D7 salivary proteins in Colombia. Front $\mathrm{Pu}$ blic Health. 2018; 6:111. Doi: https://doi.org/10.3389/ fpubh.2018.00111

12. Cantillo JF, Fernández-Caldas E, Puerta L. Immunological aspects of the immune response induced by mosquito allergens. Int Arch Allergy Immunol. 2014; 165(4):271-82. Doi: https://doi.org/10.1159/000371349

13. Marshall Js, Jawdat DM. Mast cells in innate immunity. Journal of Allergy and Clinical Immunology. 2004; 114(1):21-7. Doi: https://doi.org/10.1016/j. jaci.2004.04.045

14. Londoño-Rentería B, Cárdenas JC, Troupin A, Colpitts тм. Natural mosquito-pathogen hybrid IgG4 antibodies in vector-borne diseases: A hypothesis. Front Immunol. 2016; 7:380. Doi: https://doi.org/10.3389/ fimmu.2016.00380

15. James LK, Till sJ. Potential mechanisms for IgG4 inhibition of immediate hypersensitivity reactions. $\mathrm{Cu}$ rrent Allergy and Asthma Reports. 2016; 16:23. Doi: https://doi.org/10.1007/s11882-016-0600-2 
16. Van de Veen W, Akdis M. Role of IgG4 in IgE-mediated allergic responses. J Allergy Clin Immunol. 2016; 138(5):1434-5. Doi: https://doi.org/10.1016/j. jaci.2016.07.022

17. Furuta T, Murao LA, Lan NTP, Huy NT, Huong vTQ, Thuy TT et al. Association of mast cell-derived VEGF and proteases in dengue shock syndrome. PLOS Neglected Tropical Diseases. 2012; 6(2):e1505. https://doi. org/10.1371/journal.pntd.0001505

18. Guerrero CD, Arrieta AF, Ramirez ND, Rodríguez L-S, Vega R, Bosch I et al. High plasma levels of soluble sт2 but not its ligand IL-33 is associated with severe forms of pediatric dengue. Cytokine. 2013; 61(3):766-71. Doi: https://doi.org/10.1016/j.cyto.2012.12.024

19. Remoue F, Alix E, Cornelie S, Sokhna C, Cisse B, Doucoure $S$ et al. IgE and IgG4 antibody responses to Aedes saliva in African children. Acta Tropica. 2007; 104(2-3):108-15. Doi: https://doi.org/10.1016/j.actatropica.2007.07.011

20. Orlandi-Pradines E, Almeras L, Denis de Senneville L, Barbe S, Remoué F, Villard C et al. Antibody response against saliva antigens of Anopheles gambiae and Aedes aegypti in travellers in tropical Africa. Microbes Infect. 2007; 9(12-13):1454-62. Doi: https://doi. org/10.1016/j.micinf.2007.07.012

21. World Health Organization (wHo). Dengue guidelines for diagnosis, treatment, prevention and control: new edition [Internet]. World Health Organization. [Actualizado en 2009; citado el 18 de diciembre de 2019]. Disponible en https://apps.who.int/iris/handle/10665/44188

22. Cantillo JF, Puerta L, Lafosse-Marin S, Subiza JL, Caraballo L, Fernández-Caldas E. Identification and characterization of IgE-binding tropomyosins in Aedes aegypti. IAA. 2016; 170(1):46-56. Doi: https://doi. org/10.1159/000447298

23. Haas $H$, Tran A. Allergie aux piqûres de moustiques. Archives de Pédiatrie. 2014; 21(8):913-7.

24. Viniaker H, Lavaud F. Allergie aux piqûres de moustiques. Revue Française d'Allergologie et d'Immunologie Clinique. 2005; 45(8):620-5.
25. Van de Veen W. The role of regulatory B cells in allergen immunotherapy. Allergy and Clinical Immunology. $2017 ; 17: 447-52$.

26. Villar LÁ, Gélvez RM, Rodríguez JA, Salgado D, Parra B, Osorio L, Bosch I. Biomarcadores pronósticos de gravedad del dengue. Biomedica. 2013; 33(S1):108-16. Doi: http://dx.doi.org/10.7705/biomedica.v33i0.733

27. Avirutnan $P$, Matangkasombut P. Unmasking the role of mast cells in dengue. Elife. 2013; 2:e00767. Doi: https://doi.org/10.7554/eLife.00767

28. Singla M, Kar M, Sethi T, Kabra sk, Lodha R, Chandele A, Medigeshi GR. Immune response to dengue virus infection in pediatric patients in New Delhi, India Association of Viremia, inflammatory mediators and monocytes with disease severity. PLos Neglected Tropical Diseases. 2016; 10(4):e0004642. Doi: https://doi. org/10.1371/journal.pntd.0004497

29. Tseng Cs, Lo Hw, Teng HC, Lo wC, Ker CG. Elevated levels of plasma VEGF in patients with dengue hemorrhagic fever. FEMs Immunol Med Microbiol. 2005; 43(1):99-102. Doi: https://doi.org/10.1016/j.femsim.2004.10.004

30. Rattanamahaphoom J, Leaungwutiwong P, Limkittikul K, Kosoltanapiwat N, Srikaitkhachorn A. Activation of dengue virus-specific $\mathrm{T}$ cells modulates vascular endothelial growth factor receptor 2 expression. Asian Pac J Allergy Immunol. 2017; 35(3):171-8. Doi: https://doi.org/10.12932/AP0810

31. Ramírez-Enríquez F, Prado-Rendón J, Lachica-Valle J, Valle-Leal J. [Total immunoglobulin E as marker of allergy at Northeast of Mexico]. Rev Alerg Mex. 2016; 63(1):20-5.

32. Agüero S. Manifestaciones clínicas y bioquímicas asociadas a infección por virus del dengue en pacientes con enfermedades reumáticas durante un brote epidémico en la provincia de Catamarca, República Argentina. Rev. Arg. Reumatol. 2011; 22(3):14-28.

33. Gómez Marrugo D, Causil Garcés C, Pinzón Redondo H, Suárez Causado A, Moneriz Pretell C. Caracterización clínica del dengue en un hospital infantil de Cartagena (Colombia). Revista Salud Uninorte. 2014; 30:281-92. 